\title{
Enhanced oral absorption of 20(S)-protopanaxadiol by self-assembled liquid crystalline nanoparticles containing piperine: in vitro and in vivo studies
}

This article was published in the following Dove Press journal:

International Journal of Nanomedicine

12 February 2013

Number of times this article has been viewed

\author{
Xin Jin ${ }^{1,2}$ \\ Zhen-hai Zhang' \\ E Sun' \\ Xiao-bin Tan' \\ Song-lin $\mathrm{Li}^{3}$ \\ Xu-dong Cheng ${ }^{4}$ \\ Ming You ${ }^{4}$ \\ Xiao-bin Jia'
}

'Key Laboratory of New Drug Delivery System of Chinese Materia Medica, Jiangsu Provincial Academy of Chinese Medicine, Nanjing, People's Republic of China; ${ }^{2}$ College of Pharmacy, Nanjing University of Chinese Medicine, Nanjing, People's Republic of China; ${ }^{3}$ Department of Pharmaceutical Analysis and Metabolomics, Jiangsu Province Academy of Traditional Chinese Medicine, Nanjing, People's Republic of China; ${ }^{4}$ ALG Bioscience Co, Ltd, Suzhou, People's Republic of China

Correspondence: Xiao-bin Jia Key Laboratory of New Drug Delivery System of Chinese Materia Medica, Jiangsu Provincial Academy of Chinese Medicine, 100 Shizi Road, Nanjing, Jiangsu 210028, People's Republic of China

Tel +862585608672

$\mathrm{Fax}+862585637809$

Email xiaobinjia_nj@।26.com
Background: 20(S)-protopanaxadiol (PPD), similar to several other anticancer agents, has low oral absorption and is extensively metabolized. These factors limit the use of PPD for treatment of human diseases.

Methods: In this study, we used cubic nanoparticles containing piperine to improve the oral bioavailability of PPD and to enhance its absorption and inhibit its metabolism. Cubic nanoparticles loaded with PPD and piperine were prepared by fragmentation of glyceryl monoolein (GMO)/poloxamer 407 bulk cubic gel and verified using transmission electron microscopy and differential scanning calorimetry. We evaluated the in vitro release of PPD from these nanoparticles and its absorption across the Caco- 2 cell monolayer model, and subsequently, we examined the bioavailability and metabolism of PPD and its nanoparticles in vivo.

Results: The in vitro release of PPD from these nanoparticles was less than $5 \%$ at 12 hours. PPDcubosome and PPD-cubosome loaded with piperine (molar ratio PPD/piperine, 1:3) increased the apical to basolateral permeability values of PPD across the Caco- 2 cell monolayer from 53\% to $64 \%$, respectively. In addition, the results of a pharmacokinetic study in rats showed that the relative bioavailabilities of PPD-cubosome [area under concentration-time curve (AUC) $)_{0-\infty}$ ] and PPD-cubosome containing piperine $\left(\mathrm{AUC}_{0-\infty}\right)$ compared to that of raw $\mathrm{PPD}\left(\mathrm{AUC}_{0-\infty}\right)$ were $166 \%$ and $248 \%$, respectively.

Conclusion: The increased bioavailability of PPD-cubosome loaded with piperine is due to an increase in absorption and inhibition of metabolism of PPD by cubic nanoparticles containing piperine rather than because of improved release of PPD. The cubic nanoparticles containing piperine may be a promising oral carrier for anticancer drugs with poor oral absorption and that undergo extensive metabolism by cytochrome P450.

Keywords: 20(S)-protopanaxadiol, cubosome, piperine, Caco-2 cell monolayer, bioavailability, metabolites

\section{Introduction}

20(S)-protopanaxadiol (PPD; Figure 1) is an aglycone metabolite of PPD-containing ginsenosides (eg, $\mathrm{Rb}_{1}, \mathrm{Rb}_{2}, \mathrm{Rb}_{3}, \mathrm{Rc}, \mathrm{Rd}, \mathrm{Rg}_{3}$, and $\mathrm{Rh}$ ) after loss of the sugar moieties attached to ginsenosides in the gastrointestinal tract through a series of deglycosylation steps. ${ }^{1-3}$ PPD exhibits powerful pleiotropic anticancer effects in several cancer cell lines and inhibits metastasis. ${ }^{4-7}$ Studies have shown that among all ginsenosides, PPD has the most potent anticancer activity. ${ }^{8-11}$ PPD is currently undergoing phase I clinical trials in China. However, preclinical assessment of the pharmacokinetics of radiolabeled PPD in rats and dogs show that the oral bioavailability of PPD is low because of poor oral absorption and extensive metabolism by cytochrome P450. ${ }^{12}$ 


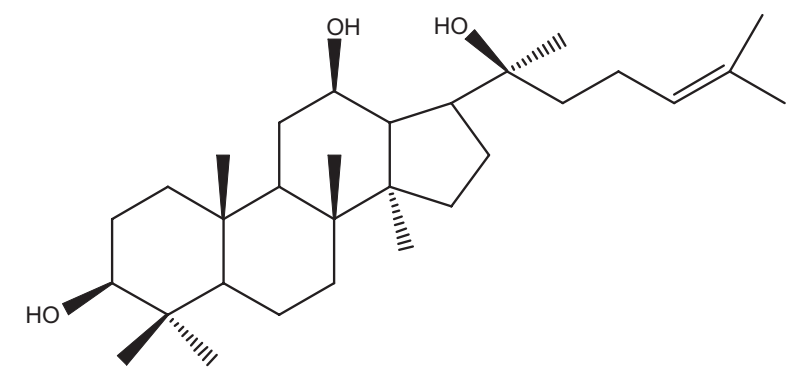

Figure I The structure of 20(S)-protopanaxadiol.

Few carriers that can help in increasing the oral bioavailability of PPD are available; ${ }^{13}$ therefore, a novel carrier that can simultaneously improve dissolution and permeation is required. Colloidal dispersions of bicontinuous cubic phases formed in a monoolein/poloxamer/water system have been proposed as potential drug carriers ${ }^{14,15}$ because (1) they are bioadhesive, (2) present a permeation enhancer as the structure-forming lipid (glyceryl monooleate), and (3) have the ability to incorporate compounds independent of their solubility and sustain their delivery. ${ }^{16-18}$ The emulsification of cubic lipid phases in water results in the formation of cubosomes and can be defined as a nanoparticle dispersion system. Dispersed particles have been shown to retain the internal structure and properties of the bulk phase. Considering the bulk of the gel, dispersions have some advantages, including large surface area and high fluidity (low viscosity). ${ }^{19}$

A previous study ${ }^{20}$ reported increased oral bioavailability of simvastatin, a Biopharmaceutics Classification System (BCS) II drug, by using glyceryl monoolein (GMO)/ poloxamer 407 cubic nanoparticles. Here, we evaluated the permeation-enhancing effect of cubic nanoparticles for their application as a potential vehicle to improve the oral bioavailability of PPD by using a more challenging BCS IV drug with poor water solubility and permeability.

Many studies have reported the pharmacokinetic interactions between several natural products. A significant beneficial consequence of these interactions is the improvement in drug bioavailability. The alkaloid piperine is a major component of black (Piper nigrum Linn) and long (Piper longum Linn) peppers. Piperine has chemopreventive effects ${ }^{21}$ and suppresses lung metastasis-induced B16F-10 melanoma in mice $^{22}$ and reduces the invasion and migration of tumor cells, ${ }^{23}$ which indicates its potential usefulness in anticancer therapy. In addition, piperine inhibits the cytochrome P450 function. ${ }^{24,25}$ Compared to the currently marketed cytochrome P450 inhibitors, piperine is safe and possesses inherent anticancer properties and thus is an ideal candidate for improving the oral bioavailability of PPD.
Many oral carriers promote absorption; however, only a few carriers are capable of both increasing absorption and inhibiting metabolism. In this study, we used PPD in combination with piperine as a self-assembled nanostructure of liquid crystalline particles in a novel drug-delivery system and evaluated the increase in its absorption.

\section{Materials and methods}

\section{Instruments and materials}

PPD (99.8\%) was purchased from the National Institute for the Control of Pharmaceutical and Biological Products (NICPBP; Beijing, People's Republic of China). GMO (RYLOTMMG19) was kindly gifted by Danisco Ingredients (Brabrand, Denmark). Poloxamer 407 (Lutrol ${ }^{\circledR}$ F127) was obtained from BASF (Ludwigshafen, Germany). Cloned Caco-2 TC7 cells were a kind gift from Dr Ming Hu of INSERM U178 (Houston, TX, USA). Piperine (99.5\%) and Hank's balanced salt solution (HBSS; powder form) were purchased from Sigma-Aldrich (St Louis, MO, USA). Milli-Q water (EMD Millipore, Billerica, MA, USA) was used throughout the study. Acetonitrile and methanol were of chromatographic grade (Merck Company Inc, Whitehouse Station, NJ, USA). All other reagents used were of analytical grade.

\section{Animal experiments}

Male Sprague-Dawley rats, weighing between $200 \mathrm{~g}$ and $250 \mathrm{~g}$, were obtained from SLEK Lab Animal Center of Shanghai (Shanghai, People's Republic of China). Animals were housed under the standard conditions of temperature, humidity, and light. Food and water were provided ad libitum. The rats were fasted overnight before the day of the experiment. All animal care measures and experimental procedures were performed according to the Guiding Principles in the Use of Animals in Toxicology, as adopted in 1989, revised in 1999, and amended in 2008, by the Society of Toxicology.

\section{Preparation of GMO-based dispersions by fragmentation}

Cubic nanoparticles were prepared by fragmentation of GMO/ poloxamer 407 bulk cubic gel. GMO and poloxamer 407 (9:1) were melted at $60^{\circ} \mathrm{C}$ in a hot water bath and then PPD with and without piperine was added and dissolved by constant stirring. Milli-Q deionized water was gradually added and the mixture was vortexed to achieve a homogenous state. After equilibration for 7 days at room temperature, an optically isotropic cubic phase gel was obtained. After the addition of deionized water, the cubic gel was disrupted by mechanical stirring. This dispersion was subsequently homogenized in 
a high-pressure homogenizer (Avestin Em-C3; Avestin, Inc, Ottawa, Canada) at 670 bar for 5 cycles. The final dispersions of the cubic nanoparticles were stored at room temperature until required.

\section{Physicochemical characterization of cubosomes}

Particle size distribution (Z-average), polydispersity index (PDI), and zeta potential of the dispersions were determined using photon correlation spectroscopy (Malvern Zetasizer 3000; Malvern Instruments Ltd, Malvern, UK). Measurements were performed at $25^{\circ} \mathrm{C}$, and the results are presented as the mean of three successive measurements of at least three independent samples. Samples were diluted with water to adjust the signal level. The morphological evaluation was performed using transmission electron microscopy (TEM; JEM-1200EX; JEOL, Tokyo, Japan).

The drug encapsulation efficiency was determined using ultrafiltration. A $1 \mathrm{~mL}$ aliquot of cubosome was transferred to a microcolumn coupled with an ultrafilter, which was then centrifuged at $4000 \mathrm{rpm}$ for 30 minutes. The amount of drug loaded in the cubosome was calculated as the difference between the total amount used in preparation of the cubosome and the amount in the filtrate determined by ultraperformance liquid chromatography (UPLC). ${ }^{31}$ The drug encapsulation efficiency was calculated as follows:

$$
\begin{aligned}
\mathrm{Q}_{\mathrm{w}}= & {\left[\left(\mathrm{W}_{\text {total }}-\mathrm{W}_{\text {free }}\right) / \mathrm{W}_{\text {total }}\right] \times 100 \% \approx\left[\left(\mathrm{C}_{\text {total }} \times \mathrm{V}_{\text {total }}\right.\right.} \\
& \left.\left.-\mathrm{C}_{\text {free }} \times \mathrm{V}_{\text {free }}\right) /\left(\mathrm{C}_{\text {total }} \times \mathrm{V}_{\text {total }}\right)\right] \times 100 \%
\end{aligned}
$$

where $\mathrm{Q}_{\mathrm{w}}$ is the drug encapsulation efficiency; $\mathrm{W}_{\text {total }}$ is the total amount of drug in the cubosome; $\mathrm{W}_{\text {free }}$ is the amount of drug in the filtrate; $\mathrm{C}_{\text {total }}$ is the concentration of drug in the cubosome; $\mathrm{C}_{\text {free }}$ is the concentration of drug in the filtrate; $\mathrm{V}_{\text {total }}$ is the volume of drug in the cubosome; and $\mathrm{V}_{\text {free }}$ is the volume of drug in the filtrate.

\section{Differential scanning calorimetry (DSC)}

Samples were sealed in aluminum crimp cells and heated at $10^{\circ} \mathrm{C} /$ minute from $30^{\circ} \mathrm{C}$ to $500^{\circ} \mathrm{C}$ in a nitrogen atmosphere
(DSC-60; Shimadzu Corporation, Kyoto, Japan). The peak transition maximum temperatures were compared using a thermal analyzer (TA-60WS; Shimadzu).

\section{Release of PPD from cubic nanoparticles}

To understand the pharmacokinetics of PPD in detail, a preliminary study on release of PPD from cubic nanoparticles of the same formulation (Table 1) was performed using the dynamic dialysis method in a ZRS-8G release tester (Tianijin tianda Tianfa Technology Co., Ltd, Tianjin, People's Republic of China). A $2 \mathrm{~mL}$ dispersion of PPD-loaded cubic nanoparticles (equivalent to $4 \mathrm{mg}$ of PPD) was sealed in dialysis bags (14,000 MWCO; Millipore) and immersed in $100 \mathrm{~mL}$ of aqueous medium containing $0.2 \%(\mathrm{w} / \mathrm{v})$ sodium lauryl sulfate (SDS) thermostatically maintained at $37^{\circ} \mathrm{C} \pm 0.5^{\circ} \mathrm{C}$ and stirred at $100 \mathrm{rpm}$. At time intervals of 1, 2, 3, 4, 6, 8, 10 , and 12 hours, $0.2 \mathrm{~mL}$ of medium was withdrawn and filtered through a $0.45 \mu \mathrm{m}$ nylon film. The PPD content was determined using UPLC as described above. Because the solubility of PPD in $0.2 \%$ SDS solution was about $0.921 \mathrm{mg} / \mathrm{mL}$, sink conditions were maintained throughout the release test. A nearly total release was observed within 1 hour for a saturated PPD solution, which indicated that the dialysis bag had a negligible effect in preventing the release of PPD.

\section{Transport experiments in the Caco-2 cell culture model}

The Caco-2 TC7 cell line was cultured in Dr Ming Hu's laboratory at INSERM U178 (Houston, TX, USA) and is similar to the wild-type Caco- 2 cells but more stable during the passage because it is a cloned cell line. ${ }^{26}$ The culture conditions for growing Caco- 2 cells were the same as described previously. ${ }^{27-30}$ Briefly, after aspirating the culture medium, the cell monolayer was washed thrice with blank HBSS (at pH 7.4 and $37^{\circ} \mathrm{C}$ ). The transepithelial electrical resistance (TEER) values of the cell monolayer were measured and were more than $350 \Omega \cdot \mathrm{cm}^{2}$. The monolayer was incubated using blank HBSS ( $\mathrm{pH}$ 7.4) for 1 hour; subsequently the incubation medium was aspirated. A solution containing the compound was then loaded onto the apical or basolateral side of the monolayer.

\begin{tabular}{|c|c|c|c|c|c|}
\hline Preparations & $\begin{array}{l}\text { Average size } \\
(\mathrm{nm})\end{array}$ & $\begin{array}{l}\text { Zeta potential } \\
(\mathrm{mV})\end{array}$ & $\begin{array}{l}\text { Polydispersity } \\
\text { index }\end{array}$ & EEa (\%) & EEb (\%) \\
\hline PPD-cubosome & $101.3 \pm 9.2$ & $-15.7 \pm 1.3$ & $0.158 \pm 0.013$ & $93.7 \pm 2.6$ & - \\
\hline $\begin{array}{l}\text { PPD-cubosome load } \\
\text { with piperine }\end{array}$ & $146.2 \pm 11.7$ & $-13.1 \pm 1.8$ & $0.188 \pm 0.024$ & $90.2 \pm 4.8$ & $92.6 \pm 3.1$ \\
\hline
\end{tabular}

Table I Physicochemical properties of 20(S)-protopanaxadiol cubosome with or without piperine

Notes: $\mathrm{n}=3$. Data represent the mean \pm standard deviation.

Abbreviations: PPD, 20(S)-protopanaxadiol; EEa, the encapsulation efficiency of 20(S)-protopanaxadiol; EEb, the encapsulation efficiency of piperine. 
The amounts of the compound transported were measured as a function of time by UPLC. Donor samples $(400 \mu \mathrm{L})$ and receiver samples $(400 \mu \mathrm{L})$ were taken at different times in triplicate and $400 \mu \mathrm{L}$ of the donor solution of the drug was added to the donor side or $400 \mu \mathrm{L}$ of blank buffer was added to the receiver side. The permeability of PPD and its preparations were compared using the same concentration $(10 \mu \mathrm{M})$ of each compound, and the samples were collected at $0,1,2,3$, and 4 hours after incubation. To each transport sample $(400 \mu \mathrm{L})$, $\mathrm{Rh}_{2}(20 \mu \mathrm{M})$ was added as an internal standard and preservative and vortexed for 30 seconds. The liquid was analyzed by UPLC after filtration. At the end of the transport experiment, the integrity of the monolayer was evaluated by measuring the TEER to ensure that there was no significant change.

\section{Oral bioavailability studies}

Male rats were randomly divided into three groups for administration of a single dose of PPD, PPD-cubosome, or PPD-cubosome containing piperine. The three groups of rats were administered oral doses equivalent to $2 \mathrm{mg} / \mathrm{kg}$ of PPD. To determine the serum drug concentrations and calculate the pharmacokinetic parameters, blood samples were collected at $0,0.25,0.5,1,1.5,2,3,4,6,8$, and 12 hours after dosing. The plasma obtained after centrifugation ( 5 minutes, $3000 \mathrm{rpm}$ ) was stored at $-20^{\circ} \mathrm{C}$ before analysis. A $100 \mu \mathrm{L}$ aliquot of the plasma sample was added to $2 \mathrm{~mL}$ polyethylene centrifuge tubes and spiked with a $10 \mu \mathrm{L}$ solution of the internal standard. After vortex-mixing for 30 seconds, $1.0 \mathrm{~mL}$ of ethyl acetate was added to each tube and the mixture was vigorously vortexed for 3 minutes. The organic and aqueous phases were separated by centrifugation at 13,000 rpm for 15 minutes. We transferred $1 \mathrm{~mL}$ of the upper organic phase to another tube and dried it by evaporating at $25^{\circ} \mathrm{C}$ using a Thermo Savant SPD 2010 Speed Vac System (Thermo Fisher Scientific, Waltham, MA, USA). The residue was dissolved in $100 \mu \mathrm{L}$ methanol and vortex-mixed for 1 minute. After centrifugation at $15,000 \mathrm{rpm}$ for 15 minutes, $20 \mu \mathrm{L}$ of the supernatant was injected into the high performance liquid chromatography-mass spectrometry system for analysis.

\section{Determination of PPD and its metabolites}

The animals were fasted for 12 hours before the experiments but had access to water. Then, a single dose of PPD, PPDcubosome, or PPD-cubosome containing piperine $(25 \mathrm{mg} / \mathrm{kg}$ ) was orally administered to the rats. Deionized water was orally administered to the rats at a dose of $10 \mathrm{~mL} / \mathrm{kg}$ body weight for blank groups. Blood samples, three as one group via the orbital sinus were collected into heparinized tubes at
2 hours after administration to three rats. Pretreatments were the same as those described above. We injected $10 \mu \mathrm{L}$ of the supernatant into the UPLC/quadrupole time-of-flight (TOF) mass spectrometry (MS) system for analysis.

\section{Chromatographic conditions and mass spectrometric conditions}

The UPLC method was used to determine the concentration of PPD in the transport samples obtained using the Caco-2 model. The UPLC conditions for PPD transport samples were as follows: system, Waters Acquity UPLC with photodiode array detector and Empower software; column, Agilent Poroshell 120 EC-C18 column (dimensions, $100 \mathrm{~mm} \times 3.0 \mathrm{~mm}$; internal diameter, $2.7 \mu \mathrm{m}$ ) (Agilent, Milford, MA, USA). The mobile phase was comprised of (A) acetonitrile and (B) aqueous solution (containing $0.1 \%$ formic acid). The UPLC elution condition was optimized as follows: gradient, 0-5 minute, $62 \%-65 \%$ A. Column temperature was maintained at $30^{\circ} \mathrm{C}$. The injection volume of the sample was $8 \mu \mathrm{L}$. The flow rate was set at $0.4 \mathrm{~mL} /$ minute. The detection wavelength was set at $203 \mathrm{~nm}$. The retention times for the internal standard and PPD were 1.91 and 3.80 minutes, respectively.

Pharmacokinetic analysis of PPD was performed using the Agilent Zorbax SB-C18 column (dimensions, $150 \mathrm{~mm} \times 2.1 \mathrm{~mm}$; internal diameter, $5 \mu \mathrm{m}$ ) at $25^{\circ} \mathrm{C}$; the mobile phase of methanol/water $(95: 5 \mathrm{v} / \mathrm{v}$, containing $0.1 \%$ formic acid) was used at a flow rate of $0.4 \mathrm{~mL} /$ minute. For atmospheric pressure chemical ionization, the probe was set at $4.0 \mathrm{kV}$, and the temperature of the probe was maintained at $350^{\circ} \mathrm{C}$. Nitrogen was used as the nebulizer gas (flow rate, $10 \mathrm{~L} /$ minute). Mass spectra were obtained at a dwell time of 0.2 and 1 second using multiple reaction monitoring. When fragment ions were detected, the masses were scanned from $\mathrm{m} / \mathrm{z} 300$ to 500. In the selective ion monitoring mode, positive ions of PPD and $\mathrm{Rh}_{2}$ were monitored at $\mathrm{m} / \mathrm{z} 425$ and 587, respectively.

Metabolites of PPD were detected using an Acquity UPLC system (Waters Corp, Milford, MA, USA) with a conditioned auto sampler at $4{ }^{\circ} \mathrm{C}$. The separation was performed using an Acquity UPLC BEH C18, $1.7 \mu \mathrm{m}, 2.1 \times 50 \mathrm{~mm}$ (Waters Corp). The column temperature was maintained at $35^{\circ} \mathrm{C}$. The analysis was achieved with gradient elution using (A) acetonitrile and (B) water (containing $0.05 \%$ formic acid) as the mobile phase. The gradient condition was: 0-1 minutes $5 \% \mathrm{~A} ; 1-1.5$ minutes, linear from $5 \%$ to $25 \%$ A; $1.5-6$ minutes, linear from $25 \%$ to $75 \%$ A; 6-12 minutes, linear from $75 \%$ to $100 \% \mathrm{~A}$; held at $100 \% \mathrm{~A}$ for 1 minute and then an immediate reduction to $10 \% \mathrm{~A}$ for equilibration of the column. The injection volume was $10 \mu \mathrm{L}$. 
The high mass resolution experiments were performed on a Synapt Q-TOF mass spectrometer (Waters Corp) equipped with an electrospray ionization source in positive ion mode. The capillary and cone voltages were 2000 and $40 \mathrm{~V}$, respectively. The desolvation gas (nitrogen) was set to $700 \mathrm{~L}$ /hour at a temperature of $350^{\circ} \mathrm{C}$, and the source temperature was $120^{\circ} \mathrm{C}$. Data were acquired from 100 to $1000 \mathrm{Da}$ and corrected during acquisition using an external reference (lock spray) consisting of a solution of $600 \mathrm{ng} / \mathrm{mL}$ leucine-enkephalin ( $\mathrm{m} / \mathrm{z}, 566.2771)$ infused at $5 \mu \mathrm{L} /$ minute. The transfer collision energy (CE) and trap CE were 4 and $6 \mathrm{eV}$ to acquire the MS data, whereas the transfer $\mathrm{CE}$ was $15 \mathrm{eV}$, and the trap CE was increased from 20 to $30 \mathrm{eV}$ to acquire the MS/MS data. The raw data were acquired and processed using MassLynx (version 4.1; Waters).

\section{Data analysis}

In the Caco-2 cell model, the rate of transport is usually obtained from the amount transported versus time curve using linear regression. Thus, the permeability of PPD was calculated using the following equation:

$$
\mathrm{P}_{\text {app }}=\frac{\mathrm{V}}{\mathrm{S} \times \mathrm{C}} \times \frac{\mathrm{dC}}{\mathrm{dt}}=\frac{1}{\mathrm{~S} \times \mathrm{C}} \times \frac{\mathrm{dM}}{\mathrm{dt}}
$$

where $\mathrm{V}$ is the volume of the receiver $(2.5 \mathrm{~mL}) ; \mathrm{S}$ is the surface area of the cell monolayer $\left(4.2 \mathrm{~cm}^{2}\right)$; and $\mathrm{C}$ is the initial concentration; hence, $\mathrm{dC} / \mathrm{dt}$ is the rate of concentration change in the receiver side and $\mathrm{dM} / \mathrm{dt}$ is the rate of drug transport. The rate of drug transport was calculated by linear regression analysis using Microsoft Excel software (Microsoft Corporation, Redmond, WA, USA).

\section{Statistical analysis}

All experiments were performed at least in triplicate. Data are presented as the mean \pm standard deviation. The data were analyzed by Student's $t$-test. A two-tailed $t$-test (Microsoft Excel) was used to determine significant differences $(P<0.05)$ compared to the controls.

\section{Results and discussion \\ Particle size, zeta potential, encapsulation efficiency, and TEM}

The mean particle diameter was $146.2 \pm 11.7 \mathrm{~nm}(\mathrm{PDI}<0.2)$ and the zeta potential was $-13.1 \pm 1.8 \mathrm{mV}$ for GMO PPDcubosome in the presence of piperine (molar ratio PPD/ piperine, 1:3; Table 1). The mean particle diameter of GMO PPD-cubosomes in the absence of piperine was $101.3 \pm 9.2 \mathrm{~nm}$
(PDI $<0.2)$ and the zeta potential was $-15.7 \pm 1.3 \mathrm{mV}$. Piperine was responsible for the difference in the mean particle diameters and zeta potentials observed between the different GMO cubosomes. Furthermore, the lipophilicity of GMO and the encapsulation efficiency of PPD and piperine in the cubosome were both above $90 \%$, which indicated that most of the drugs were encapsulated in the cubic nanoparticles.

Furthermore, we obtained the ideal particle size for GMO cubosomes; TEM showed the morphology of monodisperse cubic particles (Figure 2).

\section{DSC}

DSC was a rapid and reliable method to screen PPD, GMO, poloxamer 407, piperine, their mixtures, and the compatibility of PPD cubosome and piperine. Furthermore, this technique showed details of the maximum absorption of possible interactions. The DSC curves of PPD (a), GMO and poloxamer 407 (b), piperine (c), physical mixture (d), and PPD-cubosome loaded with piperine (e) are shown in Figure 3A. The thermogram of PPD showed two different peaks; the first peak was at $221.7^{\circ} \mathrm{C}$, while the second was at $467.9^{\circ} \mathrm{C}$. Unlike $\mathrm{GMO}$ and poloxamer 407 with no clear endothermic peaks, piperine showed a sharp endothermic peak at $147.3^{\circ} \mathrm{C}$. The physical mixture showed several peaks characteristic of PPD, GMO, poloxamer 407, and piperine; DSC of the PPD-cubosome loaded with piperine showed that the characteristic peaks of the drug had disappeared, which indicated the formation of a cubosome.

The DSC thermograms of PPD (a), GMO and poloxamer 407 (b), physical mixture (c), and the PPD-cubosome (d) are shown in Figure 3B. Thus, disappearance of the characteristic peaks of PPD in the PPD-cubosome indicate the formation of a cubosome.

\section{In vitro release}

The amount of free available PPD was about $70 \%$ at 12 hours (Figure 4). However, the overall release of PPD from the
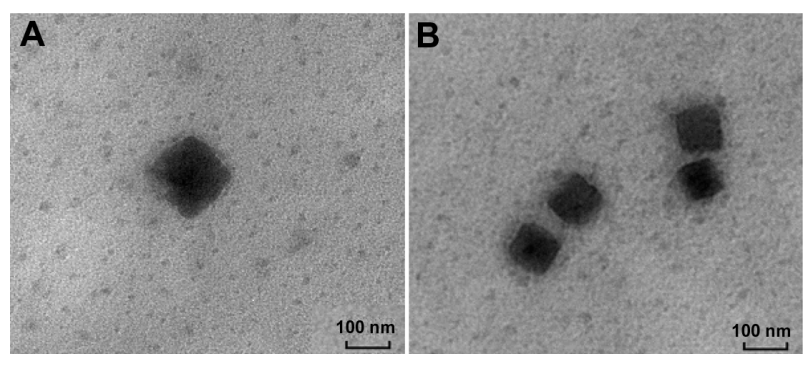

Figure 2 Transmission electron micrographs of 20(S)-protopanaxadiol cubosome with (A) or without (B) piperine. 


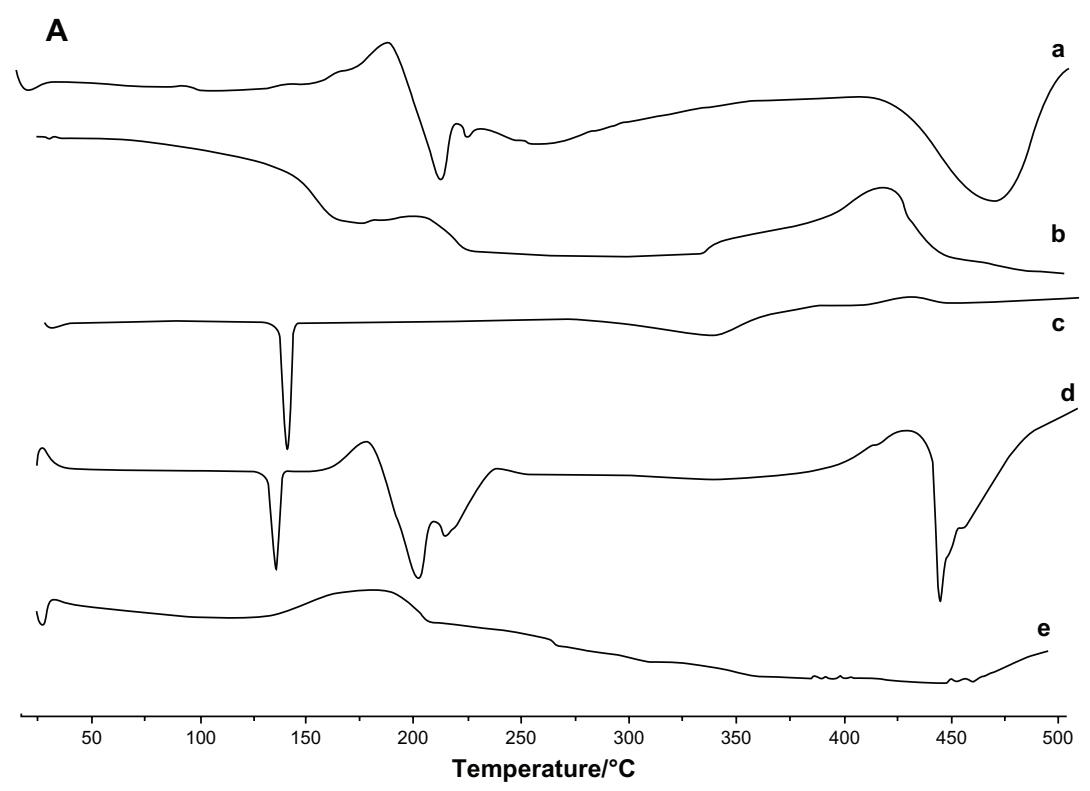

B

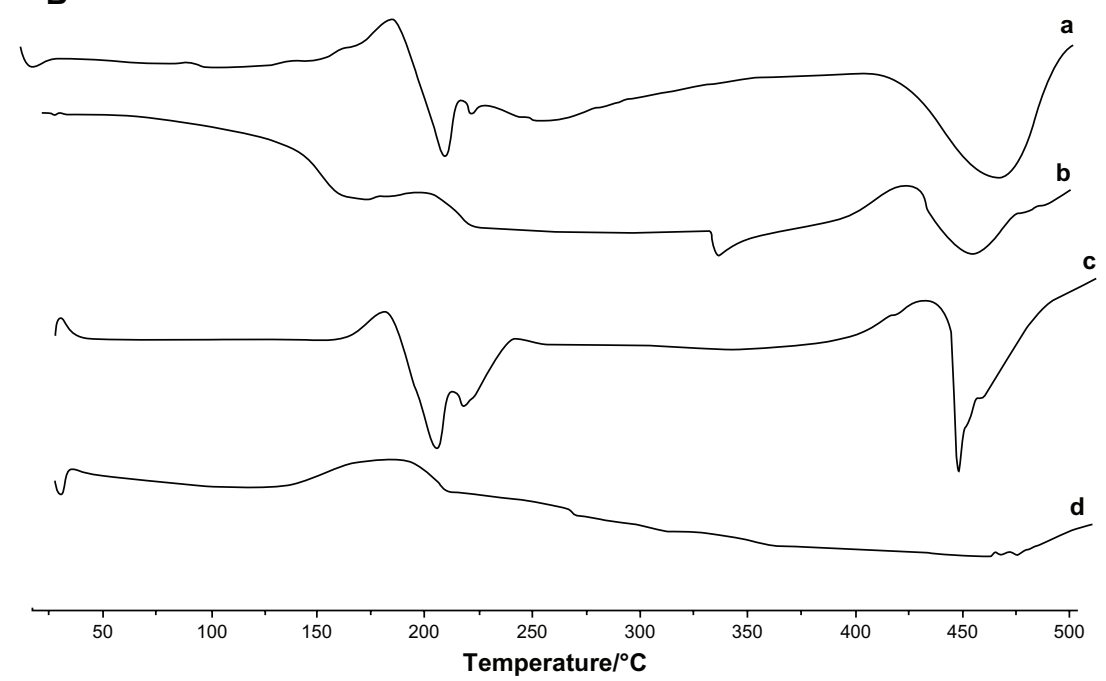

Figure 3 (A) The DSC thermograms of PPD (a), GMO and poloxamer 407 (b), piperine (c), physical mixture (d), and the PPD-cubosome load with piperine (e). (B) The DSC thermograms of PPD (a), GMO and poloxamer 407, (b) physical mixture, (c) and PPD-cubosome (d).

Abbreviations: DSC, differential scanning calorimetry; PPD, 20(S)-protopanaxadiol; GMO, glyceryl monoolein.

cubic nanoparticles was less than $5 \%$ at 12 hours. Similarly, the release of PPD from PPD-cubosome loaded with piperine was less than $5 \%$ at 12 hours. This may be because of the high affinity of PPD for the hydrophobic domain of the cubic phase that makes the release of PPD from the nanoparticles difficult. Because the overall release of PPD from the cubic nanoparticles was negligible, we did not perform extensive examination of its release from other formulations.

\section{Transport of PPD and its cubosome across the Caco-2 cell monolayer}

The Caco-2 cell culture model is routinely used to investigate drug absorption and is recognized by the Food and
Drug Administration as a viable model for human intestinal absorption. This model has been used in several previous studies on oral absorption. ${ }^{32,33}$

PPD-cubosome loaded with piperine significantly $(P<0.05)$ increased the absorption of PPD (in the apical to basolateral direction); absorptive permeability was $(1.15 \pm 0.21) \times 10^{-6} \mathrm{~cm} / \mathrm{s}$, which is higher than that of the control $(1.89 \pm 0.33) \times 10^{-6} \mathrm{~cm} / \mathrm{s}(64 \%$ increase $)$. However, the direction of transport of PPD (from the basolateral to apical side) showed no significant difference (Table 2). Compared to PPD, PPD-cubosome loaded with piperine increased absorption. This may be primarily because of the characteristics of the cubosome, which appear to increase 

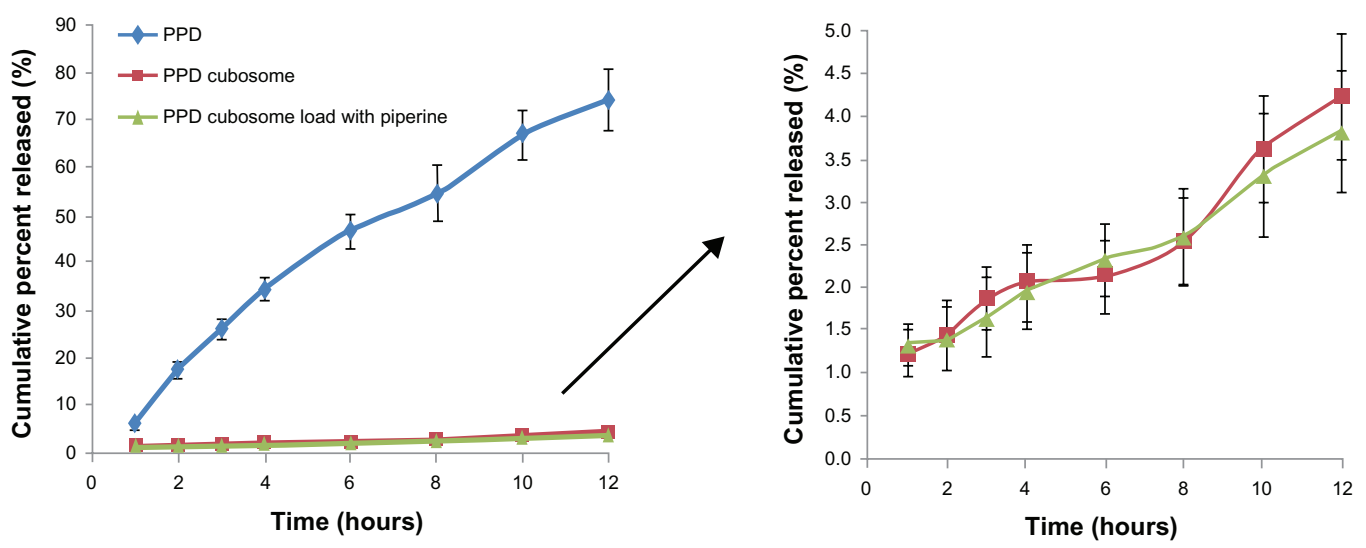

Figure 4 Release profiles of PPD, PPD-cubosome, and PPD-cubosome loaded with piperine.

Note: $\mathrm{n}=3$.

Abbreviation: PPD, 20(S)-protopanaxadiol.

bioadhesiveness and permeation by its lipid-like structure, which leads to increased membrane permeability.

Although PPD-cubosome loaded with piperine may increase the absorption of PPD by about $64 \%$, the efflux ratio of PPD-cubosome loaded with piperine was not significantly different from that of the PPD-cubosome. This may be because of fewer metabolic enzymes in the Caco- 2 cell monolayer than in the human body; thus, piperine may not have a significant impact on the efflux ratio. Furthermore, it could indicate that the limiting absorption factors are low absorption and efflux across Caco-2 cell monolayer.

\section{Pharmacokinetics of PPD and PPD- cubosome after oral administration}

A sample of PPD, PPD-cubosome, and PPD-cubosome loaded with piperine equivalent to $2 \mathrm{mg} / \mathrm{kg}$ of PPD was orally administered to rats $(n=6$; Figure 5$)$. A summary of the pharmacokinetic parameters of these three formulations is shown in Table 3; the average maximum concentration $\left(\mathrm{C}_{\max }\right)$ after oral administration of PPD was $73.45 \mathrm{ng} / \mathrm{mL}$ and the time to maximum concentration $\left(\mathrm{T}_{\max }\right)$ was about 85 minutes. However, the average value of $\mathrm{C}_{\max }$ after oral administration

Table 2 Permeabilities and efflux ratios

\begin{tabular}{|c|c|c|c|}
\hline \multirow[t]{2}{*}{ Compound } & \multicolumn{2}{|c|}{$\underline{P_{\text {app }} \times 10^{-6}(\mathrm{~cm} / \mathrm{s})}$} & \multirow{2}{*}{$\begin{array}{l}\text { Efflux } \\
\text { ratio }\end{array}$} \\
\hline & A-B & B-A & \\
\hline PPD & $1.15 \pm 0.21$ & $2.45 \pm 0.46$ & 2.13 \\
\hline PPD-cubosome & $1.76 \pm 0.25^{*}$ & $2.44 \pm 0.33$ & $1.38 *$ \\
\hline $\begin{array}{l}\text { PPD-cubosome loaded } \\
\text { with piperine }\end{array}$ & $1.89 \pm 0.33^{*}$ & $2.29 \pm 0.42$ & $1.21 *$ \\
\hline
\end{tabular}

Notes: Absorptive permeability was expressed as A-B, whereas secretory permeability was expressed as B-A. Efflux ratio was $P_{\text {app }}(B-A) / P_{\text {app }}(A-B)$. Data are presented as mean \pm standard deviation. $n=3$. $* P<0.05$ vs 20 (S)-protopanaxadiol group. Abbreviations: PPD, 20(S)-protopanaxadiol; $\mathrm{P}_{\text {app }}$, permabilities. of PPD-cubosome loaded with piperine was $142.13 \mathrm{ng} / \mathrm{mL}$ and $\mathrm{T}_{\max }$ was about 110 minutes. No significant difference was observed in the $\mathrm{T}_{\max }$ of PPD between the control group (85 minutes) and the PPD-cubosome loaded with piperine group (110 minutes). Furthermore, no significant effects were observed on the half-life in any of the groups. This may indicate that formation of PPD-cubosome loaded with piperine could increase the absorption with a delay in the release rate.

The average area under the curve from 0 to $\infty\left(\mathrm{AUC}_{0-\infty}\right)$ values of PPD and PPD-cubosome loaded with piperine in rats were 28.01 and $69.53 \mathrm{mg} / \mathrm{L} \cdot$ minutes, respectively. The $\mathrm{AUC}_{0-\infty}$ value of PPD-cubosome loaded with piperine was significantly higher than that of the controls; the $\mathrm{AUC}_{0-\infty}$ value of the PPD-cubosome loaded with piperine was 2.48 times that of PPD.

Interestingly, the $\mathrm{T}_{\max }$ values of both the PPD-cubosome and PPD-cubosome loaded with piperine were significantly high. These results were consistent with those of a previous study in which the $\mathrm{T}_{\text {max }}$ after administration in the upper small intestine was delayed by cubosome carriers. ${ }^{34,35}$ Meanwhile the clearance of PPD-cubosome and raw PPD had no significant change. Additionally, the clearance of PPD-cubosome loaded with piperine was lower than that of PPD-cubosome because of the inhibition of metabolism of PPD induced by piperine. Furthermore, the relative bioavailability of the $\mathrm{PPD}$-cubosome loaded with piperine $\left(\mathrm{AUC}_{0-\infty}\right)$ compared to that of PPD $\left(\mathrm{AUC}_{0-\infty}\right)$ and the PPD-cubosome $\left(\mathrm{AUC}_{0-\infty}\right)$ was $248 \%$ and $147 \%$, respectively.

\section{Metabolites of PPD and PPD cubosome after oral administration}

The full-scan mass spectrum of fragment ions of samples after oral administration of PPD and PPD-cubosome was 


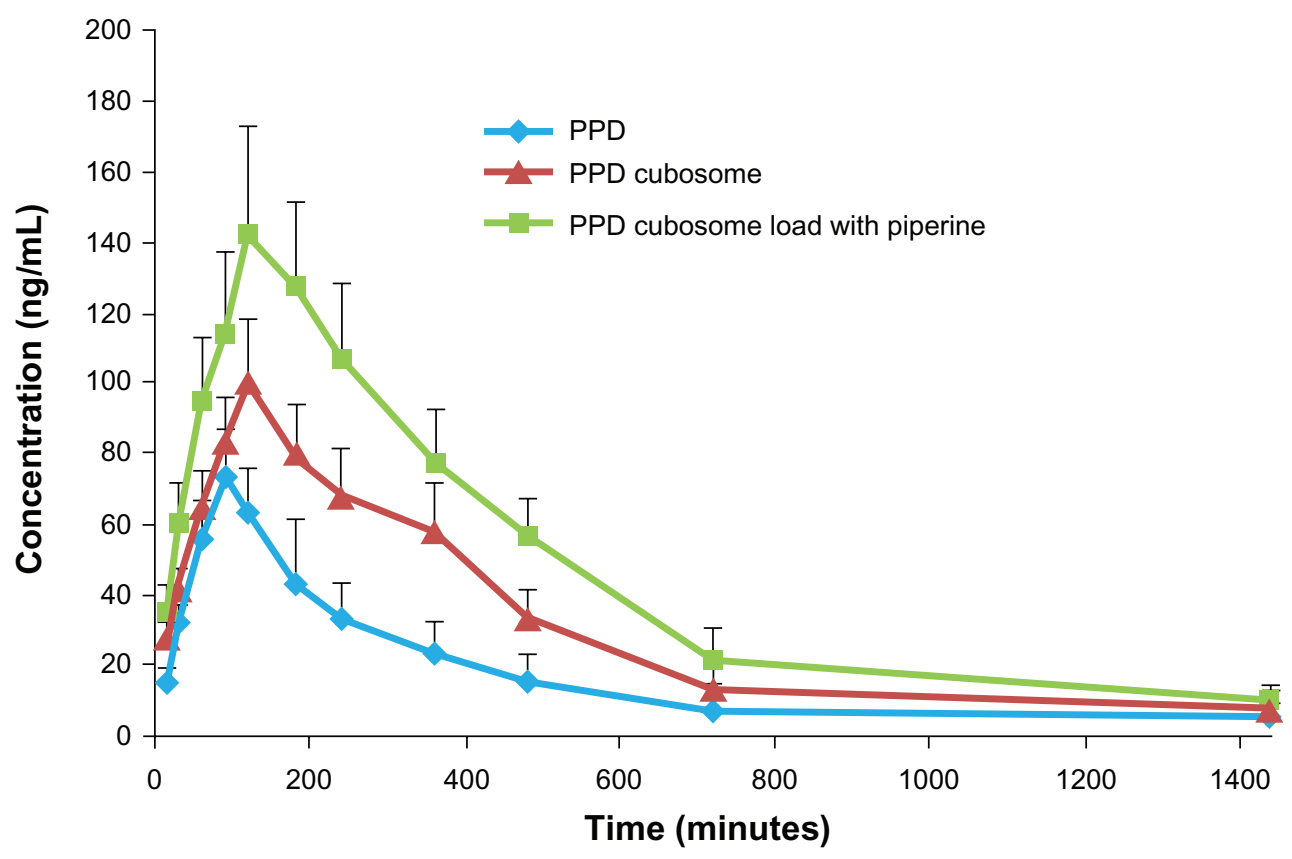

Figure 5 The plasma concentration-time curve of PPD in rats after oral administration of PPD, PPD-cubosome, and PPD-cubosome loaded with piperine (2 mg/kg, PPD). Note: Data are presented as mean \pm standard deviation $(n=6)$.

Abbreviation: PPD, 20(S)-protopanaxadiol.

compared with that of blank samples to find out the possible metabolites in rats. The abovementioned method was used to determine PPD and its seven main metabolites (M1-M7, Table 4).

UPLC/TOF-MS analysis of plasma samples showed that PPD was extensively metabolized in the plasma (Figure 6). After oral administration of PPD-cubosome, the peak area of PPD in the plasma was significantly higher than that of the controls. In addition, the metabolites of PPD were significantly increased. Thus, on the basis of these results, we can infer that PPD-cubosome increases absorption as well as metabolism. Interestingly, oral administration of PPDcubosome loaded with piperine reduced the metabolites of
PPD. Thus, PPD-cubosome loaded with piperine inhibits the metabolism of PPD.

TEM is used to characterize the internal structure of dispersed cubic particles. ${ }^{36}$ The TEM images in this study showed a typical ordered cubic texture. Furthermore, DSC may show whether most of the drug was encapsulated in the cubic nanoparticle.

The increase in the relative bioavailability of the PPDcubosome loaded with piperine compared to that of PPD and the PPD-cubosome may be because of two major factors. The in vitro study using the Caco-2 cell monolayer showed that apical to basal permeability of PPD from the cubosomes was 1.53 times (without piperine) and 1.64 times (with piperine)

Table 3 Pharmacokinetic parameters of PPD, PPD-cubosome, and PPD-cubosome loaded with piperine (2 mg/kg) administered orally to rats

\begin{tabular}{lccc}
\hline Parameters & PPD & PPD-cubosome & PPD-cubosome loaded with piperine \\
\hline $\mathrm{AUC}_{0-\mathrm{t}}\left(\mathrm{mg} / \mathrm{L}^{*} \mathrm{~min}\right)$ & $25.76 \pm 4.38$ & $43.37 \pm 6.39^{*}$ & $64.11 \pm 10.28^{*, \#}$ \\
$\mathrm{AUC}_{0-\infty}\left(\mathrm{mg} / \mathrm{L}^{*} \mathrm{~min}\right)$ & $28.01 \pm 4.02$ & $47.44 \pm 7.2 I^{*}$ & $69.53 \pm 12.76^{*, \#}$ \\
$\mathrm{MRT}_{0-\mathrm{t}}(\mathrm{min})$ & $361.18 \pm 51.43$ & $396.18 \pm 43.45^{*}$ & $407.12 \pm 52.47^{*}$ \\
$\mathrm{MRT}_{0-\infty}(\mathrm{min})$ & $489.76 \pm 63.44$ & $531.79 \pm 60.28^{*}$ & $530.17 \pm 72.33^{*}$ \\
$\mathrm{CL}(\mathrm{L} / \mathrm{min} / \mathrm{kg})$ & $0.071 \pm 0.028$ & $0.068 \pm 0.031$ & $0.029 \pm 0.01 I^{*}, \#$ \\
$\mathrm{t}_{1 / 2}(\mathrm{~min})$ & $324.01 \pm 33.49$ & $372.59 \pm 49.41$ & $345.93 \pm 45.23$ \\
$\mathrm{~T}_{\max }(\mathrm{min})$ & $85 \pm 20.61$ & $125 \pm 29.49^{*}$ & $110 \pm 14.14^{*}$ \\
$\mathrm{C}_{\max }(\mathrm{ng} / \mathrm{mL})$ & $73.45 \pm 13.56$ & $100.14 \pm 19.40^{*}$ & $142.13 \pm 31.1 I^{*, \#}$ \\
\hline
\end{tabular}

Notes: $\mathrm{n}=6$. Data represent the mean \pm standard deviation. $* P<0.05$, statistical significance: vs $2 \mathrm{mg} / \mathrm{kg} P P D ; \# P<0.05$, statistical significance: vs PPD-cubosome containing $2 \mathrm{mg} / \mathrm{kg}$ PPD.

Abbreviations: PPD, 20(S)-protopanaxadiol; AUC, area under concentration-time curve; MRT, mean residence time; $C L$, mean the clearance; $T_{\text {max }}$, time to maximum plasma concentration; $\mathrm{C}_{\max }$, maximum plasma concentration. 
Table 4 Accurate determination of the mass of metabolites of 20(S)-protopanaxadiol in rat plasma

\begin{tabular}{|c|c|c|c|c|}
\hline No & Formula & $t_{R}(\min )$ & $\begin{array}{l}\text { PPM } \\
\text { error }\end{array}$ & $M^{E}$ fragment ions \\
\hline MI & $\mathrm{C}_{30} \mathrm{H}_{52} \mathrm{O}_{5}$ & 5.10 & 3.3 & $\begin{array}{l}493.3909,475.3837 \\
457.3705,441.2263\end{array}$ \\
\hline M2 & $\mathrm{C}_{30} \mathrm{H}_{52} \mathrm{O}_{4}$ & 5.81 & 0.3 & $\begin{array}{l}\text { 477.3954, 459.3827, } \\
441.3036,143.0073\end{array}$ \\
\hline M3 & $\mathrm{C}_{30} \mathrm{H}_{52} \mathrm{O}_{4}$ & 6.60 & 2.6 & $\begin{array}{l}477.3955,459.3828 \\
441.3221,423.3622,313.2742\end{array}$ \\
\hline M4 & $\mathrm{C}_{29} \mathrm{H}_{50} \mathrm{O}_{4}$ & 9.94 & -3.7 & $\begin{array}{l}463.3770,311.2939 \\
283.2687,185.5934\end{array}$ \\
\hline M5 & $\mathrm{C}_{29} \mathrm{H}_{48} \mathrm{O}_{3}$ & 10.44 & -0.1 & $445.3677,283.266 \mathrm{I}, \mathrm{I} 83.024 \mathrm{I}$ \\
\hline M6 & $\mathrm{C}_{29} \mathrm{H}_{48} \mathrm{O}_{2}$ & 11.11 & -0.3 & $429.3731,183.0912$ \\
\hline M7 & $\mathrm{C}_{29} \mathrm{H}_{48} \mathrm{O}_{2}$ & 11.44 & -0.1 & $429.3731,183.0912$ \\
\hline PPD & $\mathrm{C}_{30} \mathrm{H}_{52} \mathrm{O}_{3}$ & 7.12 & -0.1 & $443.3902,425.3786,407.3669$ \\
\hline
\end{tabular}

Abbreviations: PPD, 20(S)-protopanaxadiol; PPM, parts per million; MSE, full information tandem mass spectrometry.

higher than that of raw PPD. Some studies have shown that the lipid bilayer of cubosomes is extremely similar to the microstructure of the cell membrane. ${ }^{37,38}$ Therefore, it is reasonable to suppose that the cubic nanoparticle-associated drugs can be transported across the epithelial membrane, thereby enhancing drug absorption. The lyotropic property enables the nanoparticles to penetrate the "unstirred water layer" and make closer contact with cell membranes. ${ }^{39-40}$ Additionally, cubic nanoparticles are isotropic, and fragmentation results in the formation of smaller cubic nanoparticles that retain the properties of their "parental" nanoparticles. Here, the secondary cubic nanoparticles or derivative vehicles like micelles may facilitate further contact with the cell membrane and increase absorption. ${ }^{41-45}$
Piperine has the potential to interact with co-administered drugs (ie, drug-herb interaction) through inhibition of metabolism of the drug mediated by cytochrome P450 enzymes. ${ }^{46}$ When the rats were treated with PPD-cubosome loaded with piperine, the cumulative absorption of PPD into the general circulation was adequately increased. This can be attributed to the ability of piperine to inhibit PPD-metabolizing enzymes, thereby circumventing first-pass metabolism. Consequently, as illustrated in the in vivo pharmacokinetic study, PPD formulated as self-assembled liquid crystalline nanoparticles (cubosomes) might exhibit increased oral bioavailability by improving the above important factors.

Therefore, multiple mechanisms rather than a single mechanism, including transportation of cubic nanoparticles across cell membranes, increases contact with cell surfaces and absorption through the process of lipid digestion and inhibits metabolism and thus may contribute to enhanced oral bioavailability of PPD using the self-assembled nanostructure of liquid crystalline particles containing a piperine carrier. Piperine, in combination with PPD-cubosome, was first reported to be highly potent in rats by increasing absorption and inhibiting metabolism. In particular, if the results of ongoing clinical trials show promising results, a combination of PPD and piperine into a cubosome carrier may actually be a superior therapeutic choice, because it could increase the oral absorption and inhibit the metabolism of PPD. Thus, new methods for increasing absorption can be created by interactions of natural products in a suitable delivery system.

However, ginsenosides are usually administered orally; subsequently, they are exposed to bacterial enzymes in the

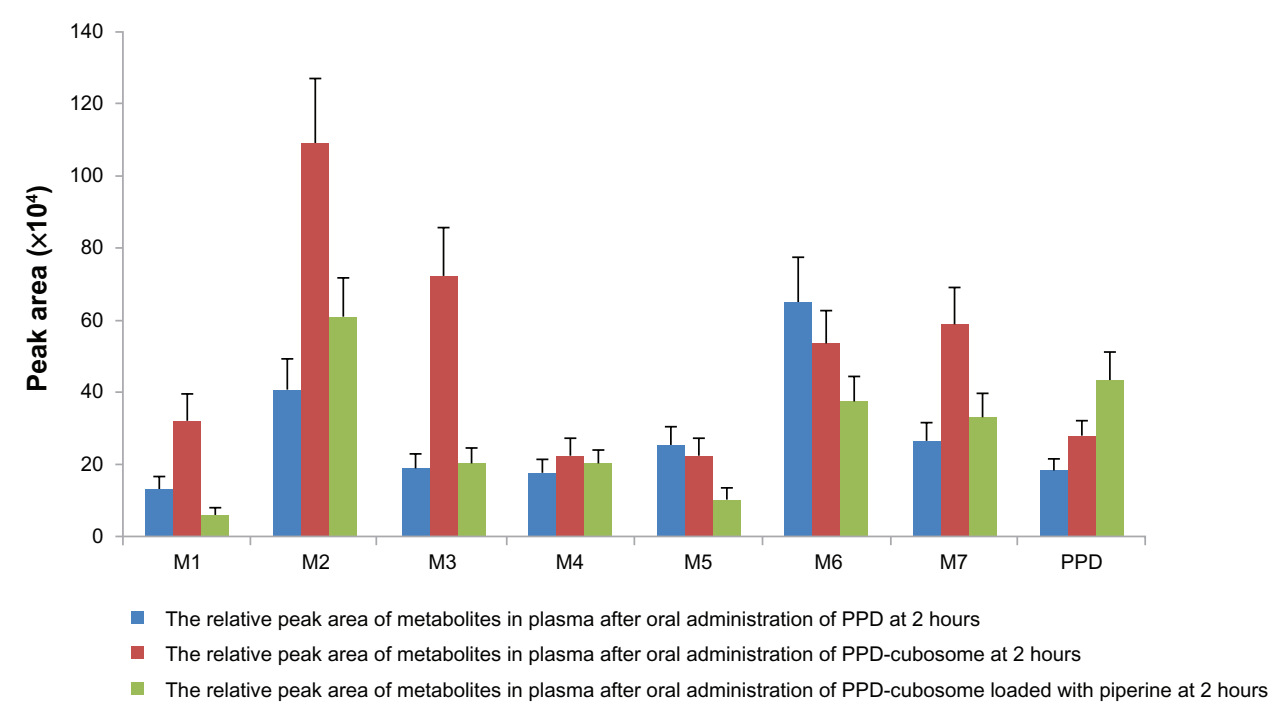

Figure 6 The relative peak area of metabolites in the plasma at 2 hours.

Note: Data are representative of three independent experiments.

Abbreviation: PPD, 20(S)-protopanaxadiol. 
gastrointestinal tract. ${ }^{47}$ The gastrointestinal tract contains more than 400 bacteria belonging mainly to the Eubacterium, Bacteroides, and Fusobactrium species and most of them are strict anaerobes. ${ }^{48,49}$ In addition, these bacteria have an excellent enzymatic system, which contributes to their enormous catalytic and hydrolytic potential. Escherichia coli and Enterococcus casseliflavus were isolated from human feces and could cleave the ring system. ${ }^{50}$ Bacteroides JY-6 and Fusobacterium K-60 produce sugars. ${ }^{51,52}$ The ginsenosides $\mathrm{Rb}_{1}, \mathrm{Rb}_{2}, \mathrm{Rc}$, and $\mathrm{Rd}$ are transformed into Compound $\mathrm{K}$ by intestinal microflora, and ginsenosides $\mathrm{Re}, \mathrm{Rg}_{1}$, and $\mathrm{Rf}$ are transformed into protopanaxatriol. ${ }^{53,54}$ Although we reviewed whether the inhibition of CYP3A4 by piperine enhances the bioavailability of PPD, we did not evaluate the metabolites of PPD produced in vitro by intestinal bacteria. In addition, it would be meaningful to study the metabolism of PPD in combination with piperine. Therefore, further studies should be performed, and they should address the metabolism of these nanoparticles containing piperine by the human intestinal bacteria to determine whether these nanoparticles also overcome the bacterial metabolism.

\section{Conclusion}

This study aimed to improve the oral bioavailability of PPD by using cubic nanoparticles to enhance absorption, and piperine was loaded for inhibition of metabolism. In the Caco-2 cell model, cubosomes loaded with piperine (molar ratio $\mathrm{PPD}$ /piperine, 1:3) increased the permeability values of PPD from the apical to the basolateral direction to about $64 \%$. Further, the results of a pharmacokinetic study in rats showed that the relative bioavailability of PPD-cubosome containing piperine $\left(\mathrm{AUC}_{0-\infty}\right)$ compared to that of raw PPD $\left(\mathrm{AUC}_{0-\infty}\right)$ was $248 \%$. In conclusion, compared to the currently marketed cytochrome $\mathrm{P} 450$ inhibitors, piperine is safe and possesses inherent anticancer properties and thus is an ideal candidate for improving the oral bioavailability of PPD. Meanwhile, a novel carrier containing piperine showed an increase in the absorption and inhibition of metabolism that is favorable for anticancer drugs that are extensively metabolized and have low absorption.

\section{Acknowledgments}

This study was financially supported by the National Natural Science Foundation of China (No 81274068), the Administration of Traditional Chinese Medicine of Jiangsu Province (LZ11066), and the open fund from Key Laboratory of New Drug Delivery System of Chinese Materia Medica in Jiangsu Province Academy of Traditional Chinese Medicine
(No 2011NDDCM02003). In addition, the foundation for high-level talent on six areas of Jiangsu province, the Suzhou city science and technology development plan in 2012 (ZXY2012009), and Key Laboratory of traditional Chinese medicine oral preparation of Chinese traditional medicine release system (2011NDDCM01001) supported this study.

\section{Disclosure}

The authors report no conflicts of interest in this work.

\section{References}

1. Bae EA, Han MJ, Kim EJ, Kim DH. Transformation of ginseng saponins to ginsenoside $\mathrm{Rh}_{2}$ by acids and human intestinal bacteria and biological activities of their transformants. Arch Pharm Res. 2004;27(1):61-67.

2. Tawab MA, Bahr U, Karas M, Wurglics M, Schubert-Zsilavecz M. Degradation of ginsenosides in humans after oral administration. Drug Metab Dispos. 2003;31(8):1065-1071.

3. Xie HT, Wang GJ, Sun JG, et al. High performance liquid chromatographic-mass spectrometric determination of ginsenoside $\mathrm{Rg} 3$ and its metabolites in rat plasma using solid-phase extraction for pharmacokinetic studies. J Chromatogr B Analyt Technol Biomed Life Sci. 2005;818(2):167-173.

4. Dong H, Bai LP, Wong VK, et al. The in vitro structure-related anti-cancer activity of ginsenosides and their derivatives. Molecules. 2011;16(12):10619-10630.

5. Li G, Wang Z, Sun Y, Liu K, Wang Z. Ginsenoside 20(S)-protopanaxadiol inhibits the proliferation and invasion of human fibrosarcoma HT1080 cells. Basic Clin Pharmacol Toxicol. 2006;98(6):588-592.

6. Liu GY, Bu X, Yan H, Jia WW. 20S-protopanaxadiol-induced programmed cell death in glioma cells through caspase-dependent and -independent pathways. $J$ Nat Prod. 2007;70(2):259-264.

7. Zhu GY, Li YW, Tse AK, et al. 20(S)-Protopanaxadiol, a metabolite of ginsenosides, induced cell apoptosis through endoplasmic reticulum stress in human hepatocarcinoma HepG2 cells. Eur J Pharmacol. 2011;668(1-2):88-98.

8. Popovich DG, Kitts DD. Structure-function relationship exists for ginsenosides in reducing cell proliferation and inducing apoptosis in the human leukemia (THP-1) cell line. Arch Biochem Biophys. 2002;406(1): $1-8$.

9. Usami Y, Liu YN, Lin AS, et al. 20(S)-protopanaxadiol and 20(S)protopanaxatriol as antiangiogenic agents and total assignment of $1 \mathrm{H}$ NMR spectra. J Nat Prod. 2008;71(3):478-481.

10. Wang W, Zhao Y, Rayburn ER, Hill DL, Wang H, Zhang R. In vitro anticancer activity and structure-activity relationships of natural products isolated from fruits of Panax ginseng. Cancer Chemother Pharmacol. 2007;59(5):589-601.

11. Yu Y, Zhou O, Hang Y, Bu X, Jia W. Antiestrogenic effect of 20Sprotopanax-adiol and its synergy with tamoxifen on breast cancer cells. Cancer. 2007;109(11):2374-2382.

12. Li L, Chen X, Li D, Zhong D. Identification of 20(S)-protopanaxadiol metabolites in human liver microsomes and human hepatocytes. Drug Metab Dispos. 2011;39(3):472-483.

13. Han MH, Chen J, Chen S, Wang X. Development of a UPLC-ESI-MS/ MS assay for 20(S)-Protopanaxadiol and pharmacokinetic application of its two formulations in rats. Anal Sci. 2010;26(7):749-753.

14. de Waard H, De Beer T, Hinrichs WL, Vervaet C, Remon JP, Frijlink HW. Controlled crystallization of the lipophilic drug fenofibrate during freeze-drying: elucidation of the mechanism by in-line raman spectroscopy. APPS J. 2010;12(4):569-575.

15. Lai J, Lu Y, Yin Z, Hu F, Wu W. Pharmacokinetics and enhanced oral bioavailability in beagle dogs of cyclosporine A encapsulated in glyceryl monooleate/poloxamer 407 cubic nanoparticles. Int J Nanomedicine. 2010;5:13-23. 
16. Garg G, Saraf S, Saraf S. Cubosomes: an overview. Biol Pharm Bull. 2007;30(2):350-353.

17. Guo C, Wang J, Cao F, Lee RJ, Zhai G. Lyotropic liquid crystal systems in drug delivery. Drug Discov Today. 2010;15(23-24):1032-1040.

18. Zeng N, Gao X, Hu Q, et al. Lipid-based liquid crystalline nanoparticles as oral drug delivery vehicles for poorly water-soluble drugs: cellular interaction and in vivo absorption. Int J Nanomed. 2012;7:3703-3718.

19. Siekmann B, Bunjes H, Koch MH, Westesen K. Preparation and structural investigations of colloidal dispersions prepared from cubic monoglyceride-water phases. Int J Pharm. 2002;244(1-2):33-43.

20. Esposito E, Eblovi N, Rasi S, et al. Lipid-based supramolecular systems for topical application: a preformulatory study. AAPS Pharm Sci. 2003;5(4):E30.

21. Vellaichamy L, Balakrishnan S, Panjamurthy K, Manoharan S, Alias LM. Chemopreventive potential of piperine in 7,12-dimethylbenz[a] anthracene-induced skin carcinogenesis in Swiss albino mice. Environ Toxicol Pharmacol. 2009;28(1):11-18.

22. Pradeep CR, Kuttan G. Piperine is a potent inhibitor of nuclear factorkappaB (NF-kappaB), c-Fos, CREB, ATF-2 and proinflammatory cytokine gene expression in B16F-10 melanoma cells. Int Immunopharmacol. 2004;4(14):1795-1803.

23. Hwang YP, Yun HJ, Kim HG, et al. Suppression of phorbol-12myristate-13-acetate-induced tumor cell invasion by piperine via the inhibition of PKC $\alpha / E R K 1 / 2$-dependent matrix metalloproteinase-9 expression. Toxicol Lett. 2011;203(1):9-19.

24. Bhardwaj RK, Glaeser H, Becquemont L, Klotz U, Gupta SK, Fromm MF. Piperine, a major constituent of black pepper, inhibits human P-glycoprotein and CYP3A4. J Pharmacol Exp Ther. 2002;302(2): 645-650.

25. Volak LP, Ghirmai S, Cashman JR, Court MH. Curcuminoids inhibit multiple human cytochromes P450, UDP-glucuronosyltransferase,an dsulfotransferase enzymes, whereas piperine is a relatively selective CYP3A4 inhibitor. Drug Metab Dispos. 2008;36(8):1594-1605.

26. Caro I, Boulenc X, Rousset M, et al. Characterisation of a newly isolated Caco-2 clone (TC-7), as a model of transport processes and biotransformation of drugs. Int J Pharm. 1995;116(2):147-158.

27. Chen J, Lin H, Hu M. Metabolism of flavonoids via entericrecycling: role of intestinal disposition. J Pharmacol Exp Ther. 2003;304(3):1228-1235.

28. Jia XB, Chen J, Lin H, Hu M. Disposition of flavonoids via enteric recycling: enzyme-transporter coupling affects metabolism of biocha$\operatorname{nin} \mathrm{A}$ and formononetin and excretion of their phase II conjugates. J Pharmacol Exp Ther. 2004;310(3):1103-1113.

29. Lv LZ, Tong CQ, Lv Q, et al. Enhanced absorption of hydroxysafflor yellow A using a self-double-emulsifying drug delivery system: in vitro and in vivo studies. Int J Nanomedicine. 2012;7:4099-4107.

30. Guan M, Zhu QL, Liu Y, et al. Uptake and transport of a novel anticancer drug-delivery system: lactosyl-norcantharidin-associated $N$-trimethyl chitosan nanoparticles across intestinal Caco-2 cell monolayers. Int $J$ Nanomedicine. 2012;7:1921-1930.

31. Gan L, Han S, Shen J, et al. Self-assembled liquid crystalline nanoparticles as a novel ophthalmic delivery system for dexamethasone: Improving preocular retention and ocular bioavailability. Int $J$ Pharm. 2010;396:179-187.

32. Coyuco JC, Liu Y, Tan BJ, Chiu GN. Functionalized carbon nanomaterials: exploring the interactions with Caco-2 cells for potential oral drug delivery. Int J Nanomedicine. 2011;6:2253-2263.

33. Wahlang B, Pawar YB, Bansal AK. Identification of permeabilityrelated hurdles in oral delivery of curcumin using the Caco-2 cell model Eur J Pharm Biopharm. 2011;77(2):275-282.

34. Nguyen TH, Hanley T, Porter CJ, Boyd BJ. Nanostructured liquid crystalline particles provide long duration sustained-release effect for a poorly water soluble drug after oral administration. $J$ Control Release. 2011;153(2):180-186.

35. Boyd BJ, Khoo SM, Whittaker DV, Davey G, Porter CJ. A lipid-based liquid crystalline matrix that provides sustained release and enhanced oral bioavailability for a model poorly water soluble drug in rats. Int $J$ Pharm. 2007;340(1-2):52-60.
36. Gustafsson J, Ljusberg-Wahren H, Almgren M, Larsson K. Submicron particles of reversed lipid phases in water stabilized by a nonionic amphiphilic polymer. Langmuir. 1997;13(26):6964-6971.

37. Lenhardt T, Vergnault G, Grenier P, Scherer D, Langguth P. Evaluation of nanosuspensions for absorption enhancement of poorly soluble drugs: in vitro transport studies across intestinal epithelial monolayers. APPS J. 2008;10(3):435-438.

38. Thomson AB, Schoeller C, Keelan M, Smith L, Clandinin MT. Lipid absorption: passing through the unstirred layers, brush-border membrane, and beyond. Can J Physiol Pharmacol. 1993;71(8):531-555.

39. Katneni K, Charman SA, Porter CJ. An evaluation of the relative roles of the unstirred water layer and receptor sink in limiting the in-vitro intestinal permeability of drug compounds of varying lipophilicity. J Pharm Pharmacol. 2008;60(10):1311-1319.

40. Korjamo T, Heikkinen AT, Mönkkönen J. Analysis of unstirred water layer in in vitro permeability experiments. J Pharm Sci. 2009;98(12): 4469-4479.

41. Angelov B, Angelova A, Papahadjopoulos-Sternberg B, et al. Detailed structure of diamond-type lipid cubic nanoparticles. J Am Chem Soc. 2006;128(17):5813-5817.

42. Angelova A, Angelov B, Papahadjopoulos-Sternberg B, Ollivon M, Bourgaux C. Proteocubosomes: nanoporous vehicles with tertiary organized fluid interfaces. Langmuir. 2005;21(9):4138-4143.

43. Angelov B, Angelova A, Papahadjopoulos-Sternberg B, Hoffmann SV, Nicolas V, Lesieur S. Protein-containing PEGylated cubosomic particles: Freeze-fracture electron microscopy and synchrotron radiation circular dichroism study. J Phys Chem B. 2012;116(26):7676-7686.

44. Angelov B, Angelova A, Mutafchieva R, et al. SAXS investigation of a cubic to a sponge (L3) phase transition in self-assembled lipid nanocarriers. Phys Chem Chem Phys. 2011;13(8):3073-3081.

45. Angelova A, Angelov B, Mutafchieva R, Lesieur S, Couvreur P. Self-assembled multicompartment liquid crystalline lipid carriers for protein, peptide, and nucleic acid drug delivery. Acc Chem Res. 2011;44(2):147-156.

46. Reen RK, Singh J. In vitro and in vivo inhibition of pulmonary cytochrome $\mathrm{P} 450$ activities by piperine, a major ingredient of piper species. Indian J Exp Biol. 1991;29(6):568-573.

47. Kong HW, Wang M, Venema K, et al. Bioconversion of red ginseng saponins in the gastro-intestinal tract in vitro model studied by highperformance liquid chromatography-high resolution Fourier transform ion cyclotron resonance mass spectrometry. J Chromatogr A. 2009; 1216(11):2195-2203.

48. Lee J, Lee E, Kim D, Lee J, Yoo J, Koh B. Studies on absorption, distribution and metabolism of ginseng in humans after oral administration. J Ethnopharmacol. 2009;122(1):143-148.

49. Lee JE, Lee S, Sung J, Ko G. Analysis of human and animal fecal microbiota for microbial source tracking. ISME J. 2011;5(2):362-365.

50. Winter J, Moore LH, Dowell VR Jr, Bokkenheuser VD. C-ring cleavage of flavonoids by human intestinal bacteria. Appl Environ Microbiol. 1989;55(5):1203-1208.

51. Jang IS, Kim DH. Purification and characterization of alpha-Lrhamnosidase from Bacteroides JY-6, a human intestinal bacterium. Biol Pharm Bull. 1996;19(12):1546-1549.

52. Kim DH, Kim SY, Park SY, Han MJ. Metabolism of quercetin by human intestinal bacteria and its relation to some biological activities. Biol Pharm Bull. 1999;22(7):749-751.

53. Tawab MA, Bahr U, Karas M, Wurglics M, Schubert-Zsilavecz M. Degradation of ginsenosides in humans after oral administration. Drug Metab Dispos. 2003;31(8):1065-1071.

54. Hasegawa H. Proof of the mysterious efficacy of ginseng: basic and clinical trials: metabolic activation of ginsenoside: deglycosylation by intestinal bacteria and esterification with fatty acid. J Pharmacol Sci. 2004;95(2):153-157. 


\section{Publish your work in this journal}

The International Journal of Nanomedicine is an international, peerreviewed journal focusing on the application of nanotechnology in diagnostics, therapeutics, and drug delivery systems throughout the biomedical field. This journal is indexed on PubMed Central, MedLine, CAS, SciSearch $\AA$, Current Contents ${ }^{\circledR} /$ Clinical Medicine,

Journal Citation Reports/Science Edition, EMBase, Scopus and the Elsevier Bibliographic databases. The manuscript management system is completely online and includes a very quick and fair peer-review system, which is all easy to use. Visit http://www.dovepress.com/ testimonials.php to read real quotes from published authors.

Submit your manuscript here: http://www.dovepress.com/international-journal-of-nanomedicine-journal 